\title{
Ionic Diffusion and Migration Fluxes in Passive Film Formation under Mixed Kinetic Control
}

\author{
Simon L. Marshall ${ }^{a}$ \\ ${ }^{a}$ CSIRO Mathematics Informatics and Statistics, Gate 5, Normanby Road, Clayton, VIC 3168, Australia \\ Email: Simon.Marshall@csiro.au
}

\begin{abstract}
The elementary processes common to all electrochemical systems are electrical conduction and the transfer of charge across the interface between two different phases. In many practically-important situations, the chemical species generated by these interfacial charge transfers can subsequently react to form poorly-conducting or insulating substances, thereby increasing the resistance and decreasing the amount of electrical energy available for reaction. This self-limiting phenomenon is commonly referred to as passivation, and is of great importance in the study of corrosion and the behavior of porous battery electrodes.
\end{abstract}

Electrochemical passivation is almost always a multistep process, involving transfer of charge across at least two interfaces and conduction phenomena taking place in the solid state and/or the electrolyte solution. It is well known that the rates of interfacial charge transfers are highly nonlinear functions of the overpotential, which is the departure of the potential difference from its equilibrium value. The transport processes may also involve nonlinearities resulting from coupling between diffusion and migration of charged species. When solid, electrically resistive reaction products are generated, the interfacial and transport phenomena are inextricably linked; there is at present no general basis on which their relative importance to the overall kinetics can be assessed. Existing treatments, based on the assumption that either process is rate-controlling, are not suitable for this purpose, since they imply that the other process is infinitely fast. What is required is a more general model allowing for mixed kinetic control, where both processes are assumed to occur at finite rates.

In this paper, the interaction between the two elementary processes identified above is explored by considering a passivation process involving deposition of a porous layer of an otherwise insulating substance. The potential and ionic concentrations within the pore electrolyte are calculated by exact solution of the steady Nernst-Planck equations, eliminating the need for the approximations inherent in existing treatments. The contributions of diffusion and ionic migration to the species fluxes are thereby determined self-consistently as a function of current density. Calculations of the electrochemical kinetic characteristics of the process lead to the conclusion that, in the presence of excess inert electrolyte, the potential drop associated with ionic conduction through the pore electrolyte makes a small contribution to the total polarization. In contrast, the surface overpotential (associated with the finite rate of interfacial charge transfer) exerts the dominant influence in the early stages of passivation, while the concentration overpotential (resulting from concentration variations within the pore electrolyte) is predominant in the later stages.

Keywords: Electrodiffusion, Nernst-Planck equation, electrochemical passivation 


\section{INTRODUCTION}

A common characteristic of the theoretical models that have been developed to describe metal passivation is the assumption that charge transport processes of some kind are rate limiting. For example, according to the Layer Pore Resistance Model (LPRM) [Müller, 1931; Calandra et al., 1974; Devilliers et al., 1986], passivation of the surface results when deposition of an insulating material reduces the volume fraction of the electrolyte in the surface layer, thereby increasing its resistance. Alternative mechanisms involve solid-state migration of ions [Cabrera and Mott, 1949; Dignam, 1972] or charged point defects [Macdonald, 1992] under the influence of an electric field, which reduces as the film thickens. Such transport phenomena are in general nonlinear, as a result of the coupling of species fluxes to each other by the potential, which satisfies Poisson's equation [Smythe, 1968].

Formation of a surface film requires transfer of charge across at least two interfaces, in addition to these conduction phenomena. The electrical behavior of such a process can be regarded as conceptually equivalent to flow of electricity through impedances in series. The simplicity of such a lumped-element representation is, however, deceptive, since each impedance depends on the current flowing through it. The contribution of each impedance to the total potential difference can be obtained by imposing a current balance condition across each interface. When the series impedances are nonlinear, this balance condition is expressed by a transcendental equation, numerical solution of which typically presents no problem. In the extension by Marshall [1993] of the LPRM to include interfacial charge transfers proceeding at a finite rate, the interfacial impedance was potential dependent, while the impedance of the solution was assumed to be constant (which is equivalent to postulating that the potential in the pore electrolyte satisfies Laplace's equation.) The applicability of that particular model is limited by the assumption that the pore electrolyte is of uniform concentration, since this implies that the migration flux of electroactive species is always sufficient to compensate for its consumption by reaction. The purpose of the present paper is to describe an improved model that is based on the solution of the steady Nernst-Planck equations for the ionic concentrations and potential, rather than the Laplace equation.

\section{Concentration and Potential Profiles}

In the absence of convection, one-dimensional transport in dilute electrolyte solutions is governed by the Nernst-Planck equations, according to which the flux $J_{i}\left(\mathrm{~mol} \cdot \mathrm{m}^{-2} \cdot \mathrm{s}^{-1}\right)$ of an ion $i$ with valence $z_{i}$, concentration $c_{i}\left(\mathrm{~mol} \cdot \mathrm{m}^{-3}\right)$, diffusivity $D_{i}\left(\mathrm{~m}^{2} \mathrm{~s}^{-1}\right)$, and mobility $u_{i}\left(\mathrm{~mol} \cdot \mathrm{s} \cdot \mathrm{kg}^{-1}\right)$ is related to the potential $\phi$ by

$J_{i}=-D_{i} \frac{\partial c_{i}}{\partial x}-z_{i} u_{i} F c_{i} \frac{\partial \phi}{\partial x}$

where $F$ is the Faraday constant $\left(96485 \mathrm{C} \cdot \mathrm{mol}^{-1}\right)$. The extra equation needed to determine the potential is either Poisson's equation $\partial^{2} \phi / \partial x^{2}=-(F / \epsilon) \sum_{i} c_{i} z_{i}$ (where $\epsilon$ is the permittivity in $\mathrm{F} \cdot \mathrm{m}^{-1}$ ), or the local electroneutrality condition $\sum_{i} c_{i} z_{i}=0$, which is an entirely adequate approximation except when the density of charge carriers is very low. We consider a three-ion system $\mathrm{M}^{+}(1), \mathrm{A}^{-}(2)$, and $\mathrm{B}^{-}(3)^{1}$, in which the concentrations in the bulk of the solution are $c_{01}, c_{02}$, and $c_{03}\left(c_{01}=c_{02}+c_{03}\right)$, and assume that the ion that is discharged is $\mathrm{A}$, according to the reaction

$\mathrm{A}^{-} \rightarrow \mathrm{A}(s)+e^{-}$

Then, introducing a reference length $L(\mathrm{~m})$, making use of the Nernst-Einstein equation $u_{i}=D_{i} / R T$ (where $R$ is the gas constant, $8.31451 \mathrm{~J} \cdot \mathrm{K}^{-1} \mathrm{~mol}^{-1}$, and $T$ is the absolute temperature, $\mathrm{K}$ ), and defining $C_{i}=c_{i} / c_{01}, X=x / L, \psi=F \phi / R T$, and dimensionless fluxes $n_{i}=J_{i} L / D_{i} c_{01}$, the flux equations become

$C_{1 X}+z_{1} C_{1} \psi_{X}=-n_{1}, \quad C_{2 X}+z_{2} C_{2} \psi_{X}=-n_{2}, \quad C_{3 X}+z_{3} C_{3} \psi_{X}=-n_{3}$.

In this case $z_{1}=1, n_{1}=0, z_{3}=-1, n_{3}=0, z_{2}=-1$, and $n_{2}=-I \equiv-\frac{i L}{F D_{2} c_{01}}$. Denoting by $r$ the ratio of the supporting electrolyte concentration to the total electrolyte concentration in the bulk solution, the vector

\footnotetext{
${ }^{1}$ The addition of an inert or supporting electrolyte to increase the conductivity is common practice in experimental electrochemistry.
} 


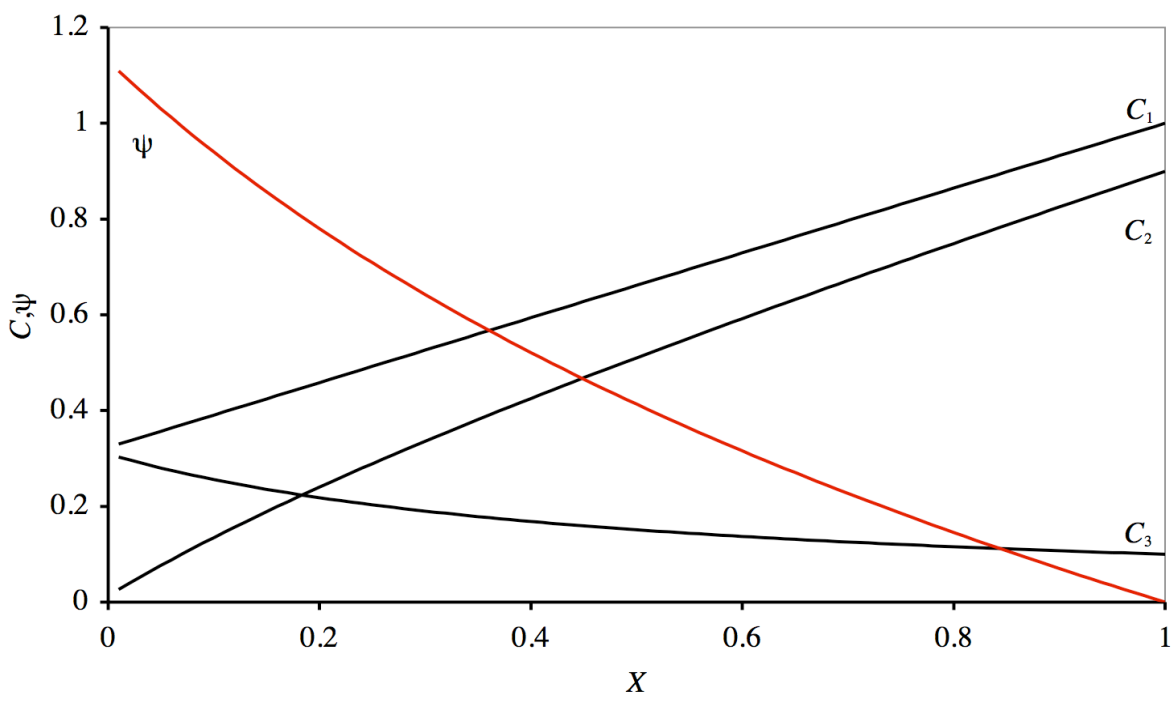

Figure 1: Ionic concentration and Potentials obtained from the Nernst-Planck equations. Electrolyte consists of cation (1), active anion (2), and inactive anion (3), with $r=0.1$. The current density is equal to 0.99 times the limiting current density.

of concentrations at the outer boundary of the layer, where $\psi=0$, is $\mathbf{C}_{0}=[1,1-r, r]^{T}$. This system of nonlinear partial differential equations can be solved as described by [Rubinstein, 1990, chapter 4], to give

$C_{1}(X)=e^{-\psi}=1-\frac{I}{2}(1-X) ; \quad C_{2}(X)=e^{-\psi}-e^{\psi} r=1-\frac{I}{2}(1-X)-\frac{r}{1+\frac{I}{2}(1-X)} ;$

and

$C_{3}(X)=e^{\psi}=\frac{r}{1+\frac{I}{2}(1-X)}$.

The current at which $C_{2}(0)=0$ is reached can be determined by solving

$0=1-\frac{I_{l i m}}{2}-\frac{r}{1-\frac{I_{l i m}}{2}} \Longrightarrow I_{l i m}=2[1-\sqrt{r}]$.

The effect of a supporting electrolyte on the corresponding potential difference across the diffusion layer can be determined by substituting this expression into that for $\psi(X)$ for $X=0$. Thus,

$\psi(0)=-\ln \left[1+\frac{I}{2}\right]=-\ln [1-1+\sqrt{r}]=-\ln \sqrt{r}$.

The form of the potential profile depends strongly on the value of $r$. For small values (e.g., $r=0.1$ ), the potential profile is nonlinear, but this curvature becomes insignificant as $r \rightarrow 1$. Deviations from linearity are imperceptible if $r \geq 0.9$, indicating that Ohm's Law is a good approximation in this case.

\section{CONCENTRATION OVERPOTENTIAL}

The variation in the composition of the layer of pore electrolyte gives rise to a concentration overpotential. For an electrode at which the the $n$-electron process

$\sum_{k} s_{k} \mathbf{M}_{k}^{z_{k}} \rightarrow n e^{-}$ 
occurs (where $s_{k}$ and $\mathrm{M}_{k}$ represent, respectively, the stoichiometric coefficient and formula of species $k$ ), this definition is expressed by [Newman, 1973, page 384]

$\eta_{c}=i_{n} \int_{0}^{\infty}\left(\frac{1}{\kappa(x)}-\frac{1}{\kappa}\right)_{\infty} d x+\frac{R T}{n F} \sum_{i} s_{i} \ln \frac{c_{i}(\infty)}{c_{i}(0)}+F \sum_{i \neq 0} \int_{0}^{\infty} \frac{z_{i} D_{i}}{\kappa(x)} \frac{\partial c_{i}(x)}{\partial x} d x$,

where $i_{n}$ is the current density normal to the electrode, and $\kappa(x), \kappa_{\infty}$ represent the conductivity at point $x$ and in the bulk of the solution, respectively. In terms of $\mathcal{D}_{2}=D_{2} / D_{1}, \mathcal{D}_{3}=D_{3} / D_{1}$, the dimensionless conductivity is

$K=\frac{R T \kappa}{c_{01} F^{2} D_{1}} \equiv \frac{\kappa}{\kappa_{0}}=C_{1}+\mathcal{D}_{2} C_{2}+\mathcal{D}_{3} C_{3}$,

which depends on $I$ and $X$ according to equations 4 . For the film formation reaction, $n=1$, and $s_{2}=1$. The dimensionless form of equation 8 is

$H_{c}=\frac{F \eta_{c}}{R T}=H_{c 1}+H_{c 2}+H_{c 3}$

where

$H_{c 1}=I \mathcal{D}_{2} \int_{0}^{1}\left(\frac{1}{K(X)}-\frac{1}{K_{\infty}}\right) d X, \quad H_{c 2}=\ln \frac{C_{i}(1)}{C_{i}(0)}, \quad H_{c 3}=\sum_{i} \int_{0}^{1} \frac{z_{i} \mathcal{D}_{i}}{K(X)} \frac{\partial C_{i}(X)}{\partial X} d X$

and $K_{\infty}=1+\mathcal{D}_{2}(1-r)+\mathcal{D}_{3} r$. The concentrations determined earlier give

$H_{c 1}=\mathcal{D}_{2}\left[\frac{1}{A} \ln \frac{A+B}{A(1-I / 2)^{2}+B}-\frac{I}{A+B}\right], \quad H_{c 2}=\ln \frac{1-r}{1-I / 2-r /(1-I / 2)}$,

and

$H_{c 3}=\frac{1}{2 A} \ln \frac{A+B}{A(1-I / 2)^{2}+B}-\mathcal{D}_{2}\left[\frac{1}{2 A}\left(1-\frac{A}{B} r\right) \ln \frac{A+B}{A(1-I / 2)^{2}+B}+\frac{r}{B} \ln \frac{1}{1-I / 2}\right]$

$-\mathcal{D}_{3} r\left[\frac{1}{2} \ln \frac{A+B}{A(1-I / 2)^{2}+B}-\ln \frac{1}{1-I / 2}\right]$,

where $A=1+\mathcal{D}_{2}$ and $B=r\left(\mathcal{D}_{3}-\mathcal{D}_{2}\right)$. The quantity $H_{c 2}$ makes the dominant contribution to $H$ as the limiting current density is approached.

\section{Surface Overpotential}

The current density $i$ for reactions of type 2 is commonly represented as

$\frac{i}{F}=k_{f} c_{2}(0) e^{\alpha F V / R T}-k_{r} e^{-(1-\alpha) F V / R T}$,

where the dimensionless parameter $\alpha \in[0,1]$ is the fraction of the total electrical energy change contributed to the activation energy of the reaction. Since the implied reversible potential $V_{r}$ satisfies $e^{F V_{r} / R T}=k_{r} /\left(k_{f} c_{2}(0)\right)$, this can be rewritten as

$\frac{i}{F}=\left[\frac{c_{2}(0)}{c_{02}}\right]^{1-\alpha} i_{0}\left(c_{02}\right)\left[e^{\alpha F \eta_{s} / R T}-e^{-(1-\alpha) F \eta_{s} / R T}\right]$

where $\eta_{s}=V-V_{r}$ is the surface overpotential and $i_{02}$ is the "true" exchange current density at bulk concentration $c_{02}$, which is a measure of the rate of the reaction in forward and reverse directions at 


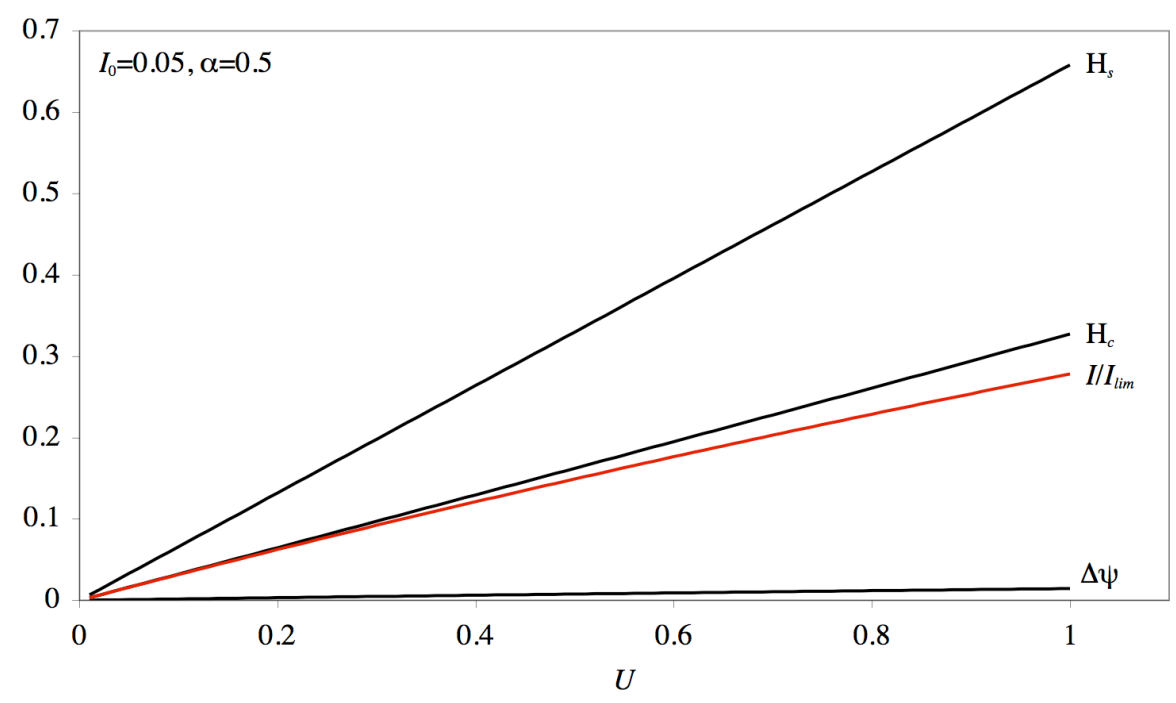

Figure 2: Contributions to total electrode potential, for Butler-Volmer kinetics with $I_{0}=0.05$ and $\alpha=0.5$, and an electrolyte with $r=0.9$.

equilibrium. This kinetic law is known as the Butler-Volmer equation. Extensive discussion of it can be found in the monograph by Newman [1973]. In dimensionless form,

$I=I_{0}\left[\frac{C_{2}(0)}{1-r}\right]^{1-\alpha}\left[e^{\alpha H_{s}}-e^{-(1-\alpha) H_{s}}\right], \quad H_{s}=\frac{F \eta_{s}}{R T}, \quad I_{0}=\frac{i_{0}\left(c_{02}\right) L}{D_{2} c_{01} F}$

The potential of the electrode (relative to the bulk solution) required to sustain a given current density is

$U \equiv \frac{F V}{R T}=\Delta \psi+H_{c}+H_{s}$

where $\Delta \psi=\psi(0)-\psi(1)$ is the potential drop across the diffusion layer. Writing this relationship as $H_{s}=U-\Delta \psi(I)-H_{c}(I)$, and observing that $\Delta \psi, H_{c}$, and $H_{s}$ are all dependent on $I$, the BulterVolmer equation defines the current density explicitly in terms of $U$, and implicitly in terms of itself. Equivalently, numerical solution of the equation

$I=\left[\frac{C_{2}(0)}{1-r}\right]^{1-a} I_{0}\left[e^{\alpha\left[U-\Delta \psi(I)-H_{c}(I)\right]}-e^{-(1-\alpha)\left[U-\Delta \psi(I)-H_{c}(I)\right]}\right]$

allows $C_{2}(0), \Delta \psi, H_{c}$, and $H_{s}$ to be determined self - consistently as a function of $U$. The results of such a calculation are shown in Figure 2, for $I_{0}=0.05$. The potential drop within the electrolyte is seen to be very small in comparison with the overpotentials.

\section{Passivation Kinetics}

When a fraction $\theta$ of the metal surface is covered by insulating film, the ionic flux equations become

$(1-\theta)\left[C_{i X}+z_{i} C_{i} \psi_{X}\right]=-n_{i}$

for each species in the pore electrolyte. For the current-carrying species (2), the equation can be written as

$C_{2 X}+z_{2} C_{2} \psi_{X}=-I /(1-\theta)$, 


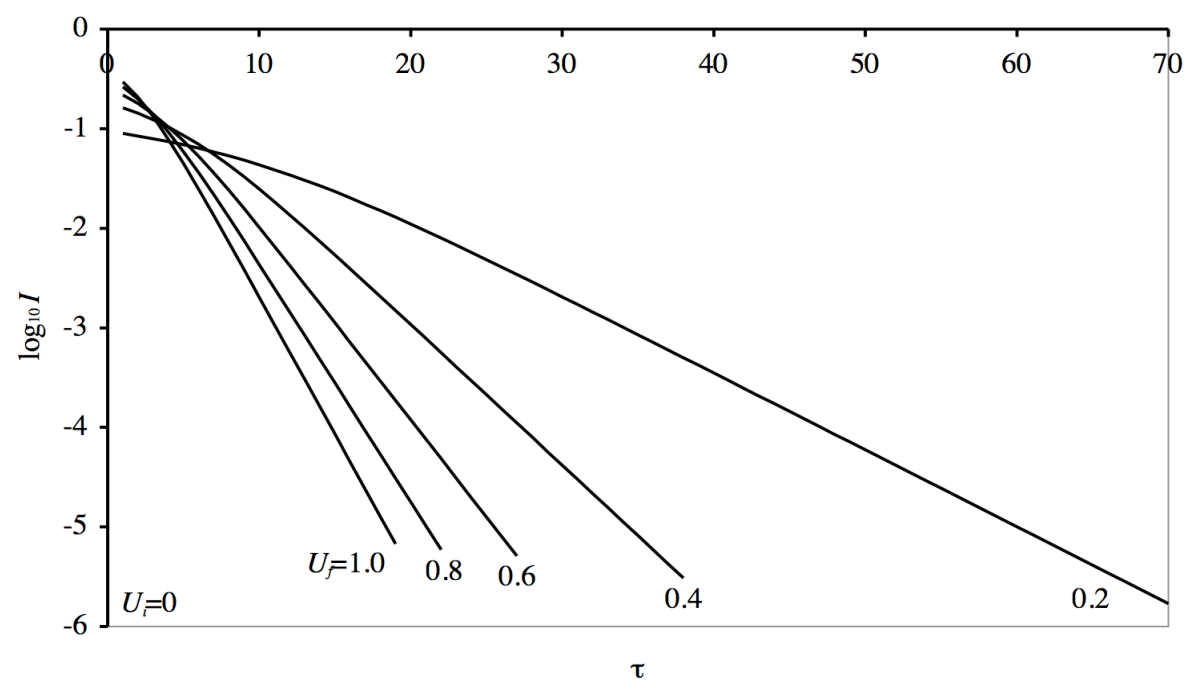

Figure 3: Transient currents resulting from potential steps from $U_{i}=0$ to different final potentials $U_{f}$. Exchange current density $I_{0}=1, r=0.9$.

and since all the other ionic fluxes are zero, the concentration distributions in the pore electrolyte are given by the earlier equations with $I$ replaced throughout by $I /(1-\theta)$. As coverage of the electrode proceeds, the implicit equation for $I$ must be solved for each value of $\theta$. In this process, the relative contributions of the different overpotentials to $U$ change. For $\theta \approx 0$, surface overpotential is dominant, and as $\theta \rightarrow$ 1 , concentration overpotential becomes dominant. In the numerical solution of the dimensionless rate equation for $\theta$, viz.,

$\frac{d \theta}{d \tau}=\left[\frac{C_{2}(0)}{1-r}\right]^{1-\alpha}\left[e^{\alpha H_{s}}-e^{-(1-\alpha) H_{s}}\right]$

(where $\tau=i_{0} t / Q_{0}$ and $Q_{0}$ is the charge density required for complete coverage). Adopting the initial condition $\theta(0)=0$, it is possible to calculate the potentiostatic transient currents resulting from an instantaneous potential steps from initial potential $U_{i}=0$ to various final potentials $U_{f}$, which are shown in Figure 3. The asymptotically log-linear form of these transients is similar to the pseudocapacitive behavior expected from the original LPRM. The transients resulting from a linear variation of potential $U=U_{0}+\sigma \tau$, are shown in Figure 4. The peak currents are found to be proportional to the 0.36 power of $\sigma$, in contrast to those calculated from the LPRM, which are proportional to $\sigma^{1 / 2}$.

\section{Discussion ANd Conclusions}

The foregoing treatment of electrode passivation is notable in being entirely nonlinear, but requiring no additional approximations. The exact solution of the system of nonlinear coupled PDEs defining the steady potential and concentration profiles eliminates the need for the assumption that the electric field is constant. The calculations presented show that, far from being negligible (as assumed in the LPRM), the surface and concentration overpotentials make highly significant contributions to the total polarization. In the presence of a large excess of inert supporting electrolyte, these overpotentials are much larger than the potential drop within the pore electrolyte, but in the case of a binary electrolyte (for which $r=0$ ), equation 6 shows that the solution potential drop could also become very large. The conclusion reached in the earlier work [Marshall, 1993] that unrealistically high exchange current densities would be required for the assumptions of the original LPRM to be justified is also valid for the present treatment. But the divergence of the logarithmic term $H_{c 2}$ in equation 11 as $C_{2}(0) \rightarrow 0$ ensures that the concentration overpotential will always become dominant as the limiting current density (which decreases as $\theta \rightarrow 1$ ) is approached. 


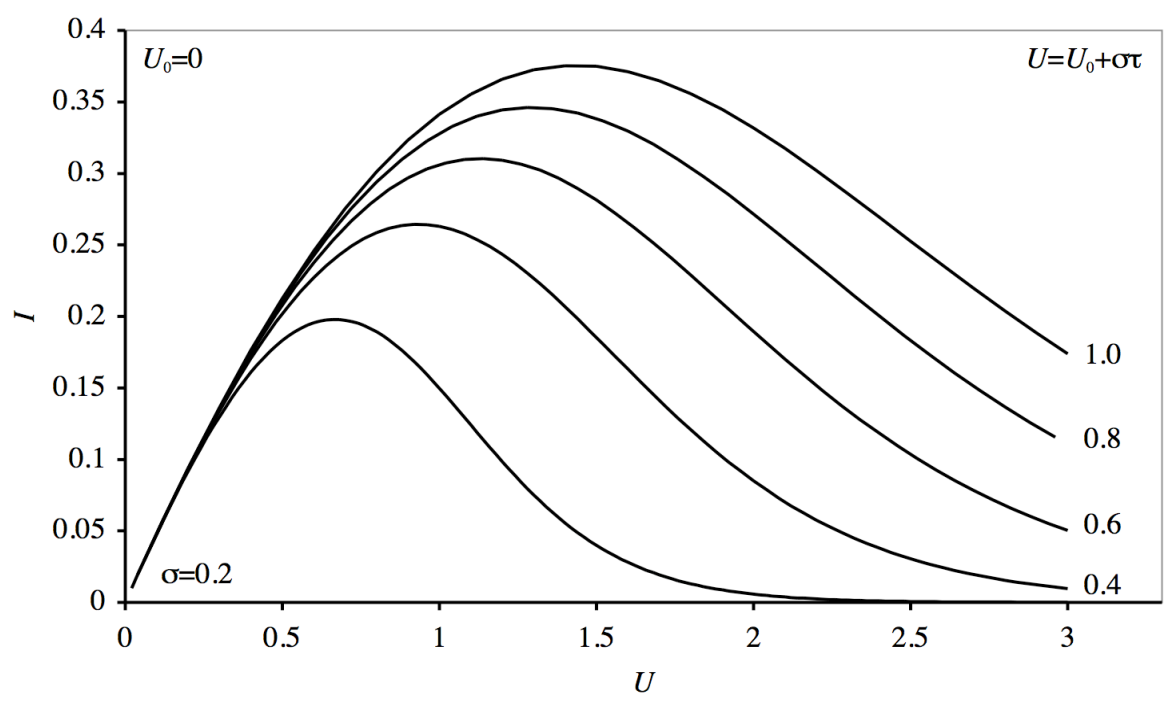

Figure 4: Transient currents resulting from linear potential scans from initial potential $U_{0}=0$, at different dimensionless scan rates $\sigma$. Exchange current density $I_{0}=1, r=0.9$.

This means that a situation in which the resistance of the electrolyte overpowers the combined effects of concentration and surface overpotentials is even less likely to be practically realizable.

\section{ACKnOWLedgments}

I have pleasure in thanking the anonymous referees for their helpful comments. Financial support from the Wealth From Oceans Flagship is gratefully acknowledged.

\section{REFERENCES}

Cabrera, N. and N. Mott (1949). Theory of the oxidation of metals. Reports on Progress in Physics 12, 163-185.

Calandra, A., N. de Tacconi, and A. Arvía (1974). Potentiodynamic current/potential relations for film formation under ohmic resistance control. Electrochimica Acta 19(12), 901-905.

Devilliers, D., F. Lantelme, and M. Chemla (1986). Surface processes: effect of ohmic polarization on potentiodynamic V/I curves. Electrochimica Acta 31(10), 1235-1245.

Dignam, M. (1972). Mechanisms of ionic transport through oxide films. In J. W. Diggle (Ed.), Oxides and Oxide Films, Volume 1, Chapter 2, pp. 91-537. New York: Marcel Dekker.

Macdonald, D. D. (1992). The point defect model for the passive state. Journal of the Electrochemical Society 139(12), 3434-3449.

Marshall, S. L. (1993). Analysis of kinetic and ohmic polarization effects in passivation reactions under potentiodynamic control. Electrochimica Acta 38, 2699-2706.

Müller, W. (1931). On the passivity of metals. Transactions of the Faraday Society 27, 737-751.

Newman, J. S. (1973). Electrochemical Systems. Prentice-Hall International Series in the Physical and Chemical Engineering Sciences. Englewood Cliffs, NJ: Prentice-Hall.

Rubinstein, I. (1990). Electro-Diffusion of Ions, Volume 11 of SIAM Studies in Applied Mathematics. Philadelphia, PA: Society for Industrial and Applied Mathematics.

Smythe, W. R. (1968). Static and Dynamic Electricity (3rd ed.). New York: McGraw-Hill. 